\title{
Tres nuevos registros de asteroideos (Echinodermata: Asteroidea) de Perú
}

\section{Three new records of asteroids (Echinodermata: Asteroidea) from Peru}

\author{
Yuri Hooker ${ }^{1,2}$ y Francisco A. Solís-Marín ${ }^{3}$
}

\begin{abstract}
(1) Laboratorio de Biología Marina, Facultad de Ciencias y Fisiología, Universidad Peruana Cayetano Heredia. Av. Honorio Delgado 430, Urb. Ingeniería, S.M.P. Lima - Perú. Email: hookery@yahoo.com

(2) Unidad Marino Costera, Servicio Nacional de Áreas Naturales Protegidas por el Estado (SERNANP) Ministerio del Ambiente, Perú

(3) Colección Nacional de Equinodermos, Instituto de Ciencias del Mar y Limnología, Universidad Nacional Autónoma de México.
\end{abstract} Aceptado: $\quad 16 / 09 / 2011$ Publicado online: $08 / 02 / 2012$

\begin{abstract}
Resumen
En el presente trabajo se registran 3 nuevos asteroideos (Echinodermata: Asteroidea) de aguas someras (4 - >50 m) para el Perú: Astropecten regalis Gray, 1840, Paulia horrida Gray 1840 y Meyenaster gelatinosus (Meyen, 1834). Astropecten regalis se conocía desde el Golfo de California hasta Panamá, en el presente trabajo, se amplía su distribución hasta Máncora, Perú. La distribución geográfica de Paulia horrida era conocida desde Baja California, hasta Isla Cocos, Costa Rica, en este estudio se amplía su distribución geográfica hasta Punta Sal, Perú. A Meyenaster gelatinosus se le conocía solo de Chile, en el presente trabajo se registra y confirma su presencia en el Perú, ampliando su distribución norte hasta San Juan de Marcona. Se proporciona información morfológica de las especies, características del hábitat y fotografías in situ y de los especímenes recién recolectados.
\end{abstract}

Palabras clave: nuevos registros, estrellas de mar, Perú.

\section{Abstract}

The aim of this work is to present three new shallow water $(4->50 \mathrm{~m})$ records of asteroids (Echinodermata: Asteroidea) for the Peruvian fauna: Astropecten regalis Gray, 1840, Paulia horrida Gray 1840 and Meyenaster gelatinosus (Meyen, 1834). Astropecten regalis geographical distribution is known that ranges from Gulf of California to Panama, this discovery extends its distribution to Mancora, Peru. Paulia horrida is known from Baja California to Isla Cocos, Costa Rica, and this record extends its southern distribution limit to Punta Sal, Peru. Meyenaster gelatinosus was considered endemic to Chilean waters, however, this record confirm its presence in Peru extending its northern distribution limit to San Juan de Marcona, Peru. Morphological and habitat information on this four species is provided, together with live pictures.

Keywords: new records, sea star, Peru.

\section{Introducción}

Los equinodermos, a pesar de su gran importancia ecológica, son un grupo poco estudiado en el Perú. Los trabajos de Clark (1910), Madsen (1956), Hooker et al. (2005), Paredes y Gamarra (2006) y Prieto (2010) son publicaciones donde se tratan a los equinodermos del Perú desde el punto de vista taxonómico y ecológico. Otros autores han registrado algunas especies recolectadas en aguas peruanas durante cruceros o investigaciones en áreas geográficas amplias (Aggassiz 1881, Deichmann 1941, 1958, Bluhm \& Gebruk 1999).

El conocimiento de la diversidad de estrella de mar en un ecosistema o área geográfica es de relevancia pues son especies clave en las comunidades tanto de sustratos duros como blandos y en toda la gradiente batimétrica de los océanos. Howell et al. (2003) los clasifica en 3 grupos tróficos: suspensívoros, depredadores/carrońeros y sedimentívoros. La mayoría de estrellas de mar son carnívoras y pueden ser los mayores depredadores en algunos hábitats (Gil \& Zaixso 2008, Himmelman et al. 2005).

Los nuevos registros de asteroideos para el Perú se constituyen como un importante aporte al conocimiento de la biodiversidad de equinodermos del mar peruano y componentes importantes de la estructura comunitaria a las que pertenecen, contribuyendo además a la mejor comprensión biogeográfica del Pacífico Oriental tropical y del Pacífico Sur Oriental templado.

\section{Material y métodos}

Se realizaron colectas directas por medio de buceo SCUBA y utilizando artes de pesca. Los especímenes fueron fotografiados in situ, tomando el dato batimétrico por medio de un computador de buceo. La recolecta fue directa, transportándose los especímenes vivos hasta el laboratorio de campo. Un espécimen de Astropecten regalis fue colectado con red de arrastre de playa (chinchorro) y otro fue encontrado varado en la playa. Todos los especímenes, excepto Paulia horrida, fueron fijados en formalina neutralizada por 24 horas, enjuagados y desecados al medio ambiente y posteriormente en estufa. $P$. horrida fue directamente fijada en alcohol al $96 \%$ y conservada de manera permanente en alcohol al 70\%. Los especímenes se encuentran depositados en la Colección de Zoología Acuática (CZA) de la Universidad Peruana Cayetano Heredia y en IMARPE. La medida R o "radio mayor" proporcionada fue tomada desde el centro del disco del espécimen hasta la punta del brazo más largo.

Todos los especímenes fueron recolectados por el autor, excepto un ejemplar de Meyenaster gelatinosus que fue recolectado por el Blgo. Marco Rios.

\section{Resultados}

Se registra por primera vez para el Perú a Meyenaster gelatinosus (Meyen, 1834), Paulia horrida Gray 1840, Astropecten regalis Gray, 1840 y Luidia superba Clark, 1917.

\section{Clase Asteroidea \\ Orden Paxillosida Perrier, 1884 \\ FAMILIA AstropectinIDAE Gray, 1840}

\section{Astropecten regalis Gray, 1840}

Figura 1

Material examinado: 2 especímenes: 1 espécimen (CZM58), $\mathrm{R}=51 \mathrm{~mm}$, recolectado en Isla del Amor, manglares de tumbes $\left(3^{\circ} 29^{\prime} 20,67^{\prime \prime} \mathrm{S}-80^{\circ} 23^{\prime} 14,85^{\prime \prime W}\right)$ y 1 espécimen (CZM- 


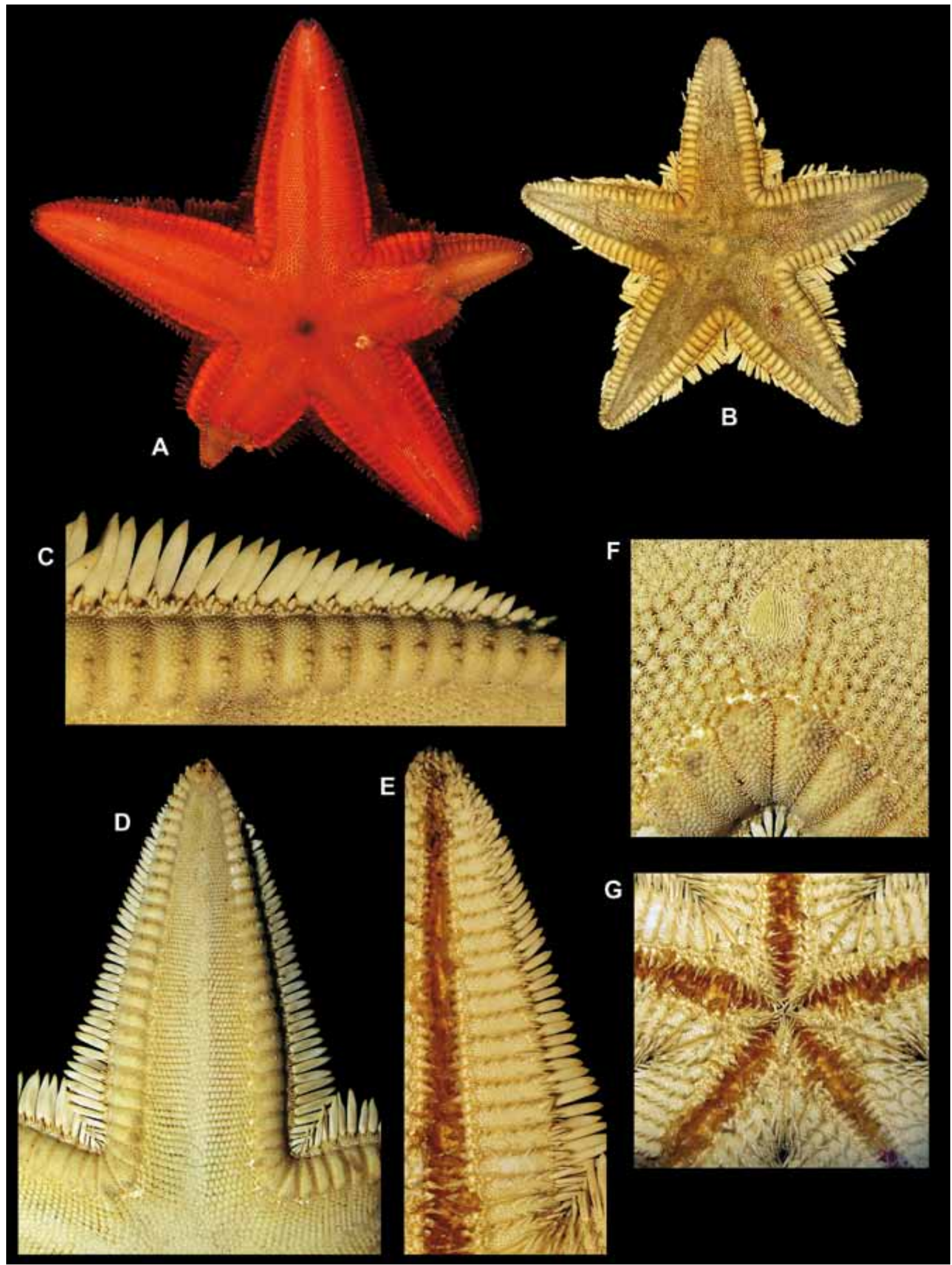

Figura 1. Astropecten regalis: A. Espécimen vivo en regeneración (CZM-58); B. Espécimen desecado (CZM-59); C. Placas súperomarginales; D. Superficie abactinal del brazo; E. Placas ínferomarginales y surco ambulacral; F. Madreporito rodeado de espinas paxilares; G. Boca, espinas orales y ambulacros. 
59), $\mathrm{R}=53 \mathrm{~mm}$, encontrado varado en Máncora (46'36,95”S $-81^{\circ} 3$ '55,60”W).

Caracteres diagnósticos: Cuerpo plano, brazos cortos y anchos, ligeramente constrictos hacia la base, ensanchándose ligeramente y luego adelgazándose homogéneamente hasta el extremo. Discos y brazos cubiertos completamente por espinas paxilares romas, con 1 a 8 gránulos en la parte central y rodeada por más de 12 espinillas marginales. Placas súperomarginales cubiertas por gránulos redondos, varios de ellos más grandes, formando una fila. Placas ínferomarginales planas, cubiertas por gránulos planos, y a uno de sus lados y en el margen externo, una fila de espinas aplanadas. Borde de las placas marginales con grandes espinas planas con puntas aguzadas. Madreporita esférica, con tabiques longitudinales.

Color: Anaranjado intenso en vida, especímenes secos amarillentos.

Hábitat: En fondo arenoso, cerca del intermareal. Se le menciona como fauna acompañante de la pesca de arrastre de langostino en Perú (hasta unos $50 \mathrm{~m}$ de profundidad).

Distribución: Conocida del Golfo de California hasta Panamá (Caso 1979). Se amplía su distribución hasta Máncora, Perú.

Orden Valvatida Perrier, 1884

FAMILIA AsterodiscididAe Rowe, 1977

\section{Paulia horrida Gray 1840}

Figura 2

Material examinado: 1 espécimen (CZM-62) R= $84 \mathrm{~mm}$, recolectado en Punta Sal (03056'32"S - 8056'46,3”O) a 33 $m$ de profundidad.

Caracteres diagnósticos: Disco bien desarrollado y poco elevado, región ventral aplanada; con 5 brazos anchos, triangulares. Fuertes espinas cónicas cubren la superficie abactinal (aboral), las placas abactinales con numerosos poros a través de los cuales salen las pápulas. Superficie actinal con placas actinales teseladas, cada una con una protuberancia rodeada por pequeños gránulos. Placas súperomarginales con grandes espinas esféricas distintivas de la especie (Figura 3B). Surco ambulacral estrecho, las placas interambulacrales tienen una hilera de espinas internas pequeńas, digitiformes, varias en cada placa, y una serie externa de espinas más grandes, dos en cada placa.

Color: en vida es rojo bermellón intenso.

Hábitat: se le ha encontrado sobre un arrecife rocoso a $33 \mathrm{~m}$ de profundidad, con abundantes pólipos de corales ahermatípicos y depósitos de sedimento orgánico.

Distribución: Se le conocía de Punta Santa Elena, Ecuador y en las Islas Galápagos (Clark 1910), también en Todos Santos (Baja California), Isla Cocos e Isla Clarión (A. M. Clark 1993), se amplía su distribución hasta Punta Sal, Perú.

Orden Forcipulatida Perrier, 1884

FAMILIA AsteriIdae Gray, 1840

Meyenaster gelatinosus (Meyen, 1834)
Material examinado: 2 especímenes: 1 espécimen $(\mathrm{UPCH}$ CZM-56) $\mathrm{R}=141 \mathrm{~mm}$, recolectado el 5 de diciembre del 2008 en el islote El Avión, San Juan de Marcona (15²3’26,02”S $\left.75^{\circ} 10^{\prime} 45,02^{\prime \prime W}\right)$ a $19 \mathrm{~m}$ de profundidad; 1 espécimen, $\mathrm{R}=185$ $\mathrm{mm}$, recolectado en Punta Picata, Tacna, en el 2006 (Recolector: Marco Ríos).

Caracteres diagnósticos: 5 o 6 brazos (comúnmente 6). Esqueleto abactinal (aboral) reticulado, poco calcificaco. Brazos con una fila de espinas carinales a lo largo del radio y dos filas de espinas marginales, todas bien desarrolladas. Espinas rodeadas de grandes cojines de pedicelarios, abundantes y minúsculos. Poros papulares grandes, reunidos en grupos; pápulas bien desarrolladas.

Color: blanco amarillento en vida, también desecadas. Un juvenil observado presentó color marrón-ocre.

Hábitat: sobre fondos rocosos y aguas bien oxigenadas. Se le ha observado entre 4 y 19 m de profundidad.

Distribución: Anteriormente solo conocida en Chile, sin embargo H. L. Clark (1910) suponía su presencia en el sur del Perú. En el presente trabajo se registra y confirma su presencia en el Perú, ampliando su distribución norte hasta San Juan de Marcona.

\section{Observaciones}

Mutschke y Mah (2009) indican que Meyenaster gelatinosus se encuentra en Perú basándose en la publicación de Madsen (1956). Al revisar la citada publicación, Perú no se menciona dentro del rango de distribución de la especie, sin embargo en un gráfico de grupos faunísticos de la misma publicación, se incluye erróneamente a $M$. gelatinosus en Perú lo que puede haber generado la confusión. Este registro no se considera válido.

En la publicación de H. L. Clark (1910) "The Echinoderms of Peru”, a pesar del título, hay algunas especies que no las registra para Perú y solo supone su presencia en este país. Ese el es caso de Paulia horrrida Rowe (1977) menciona a Perú dentro de las localidades de registro de la especie, muy probablemente basándose en la publicación de H. L. Clark (1910), ya que no menciona haber revisado algún espécimen recolectado en aguas peruanas. Además, actualmente no se conoce ningún espécimen de $P$. horrida recolectado en Perú y depositado en alguna colección científica, por lo que suponemos que este es el primer ejemplar realmente registrado para Perú. El registro tiene además el valor de haber obtenido, hasta donde sabemos, la primera fotografía de esta especie en su hábitat natural.

Los hallazgos mencionados en el presente trabajo son parte de los esfuerzos que se vienen realizando por inventariar la diversidad acuática de las áreas marinas protegidas del Perú así como para identificar y priorizar localidades con alta biodiversidad que deben ser protegidas dentro del Sistema Nacional de Áreas Naturales Protegidas, siendo Punta Sal, localidad de colecta de Paulia horrida, uno de los lugares que ya se encuentra propuesto para su protección.

\section{Literatura citada}

Agassiz A. 1881. Report of the Echinoidea dredged by the H.M.S. Challenger during the year 1873-1876. Zool. 3(9): 1-321.

Bluhm H. \& A. Gebruk. 1999. Holothuroidea (Echinodermata) of the Peru Basin - Ecological and Taxonomic Remarks Based on Underwater Images. Mar.Ecol., 20(2): 167-195. 


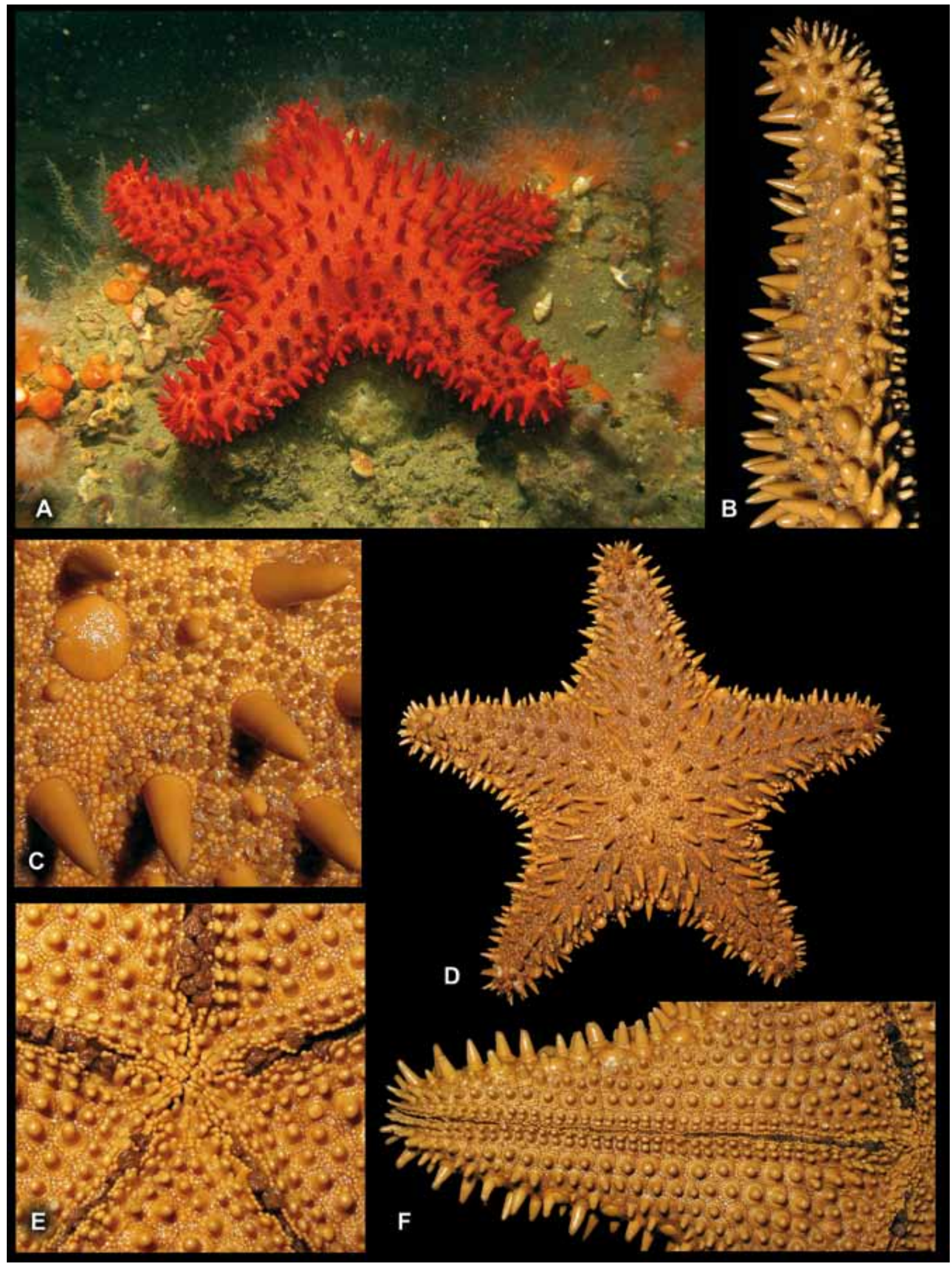

Figura 2. Paulia horrida: A. Espécimen in situ; B. Espinas superomarginales esféricas; C. Madreporito, espinas abactinales y pápulas; D. Espécimen desecado; E. Boca, ambulacros y espinas interambulacrales ; F. Superficie actinal. 


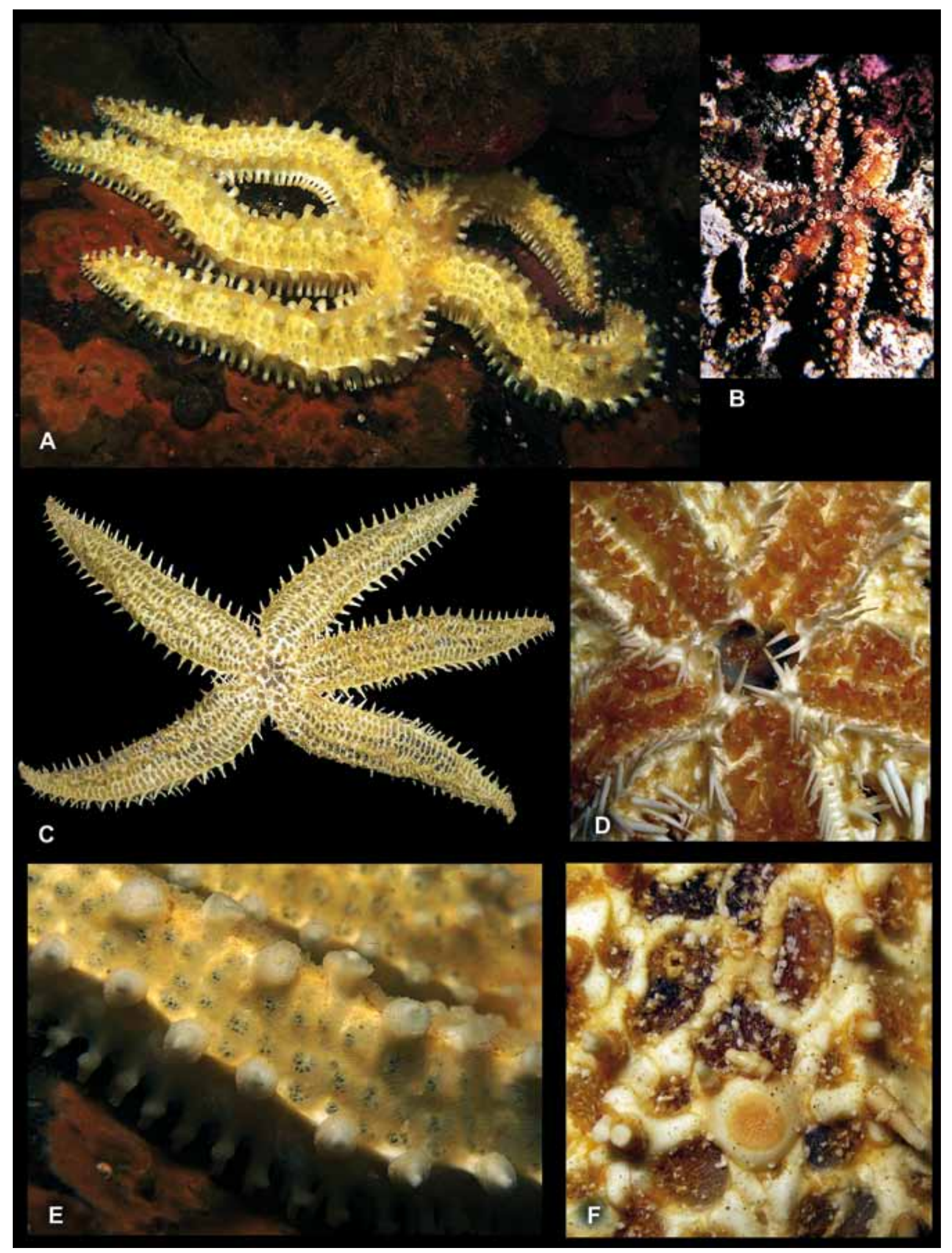

Figura 3. Meyenaster gelatinosus: A. Adulto in situ; B. Juvenil in situ; C. Espécimen desecado; D. Boca, espinas orales y surcos ambulacrales; E. Espinas carinales y marginales rodeadas de cogines de pedicelarios, se observa poros y pápulas; F. Madreporito. 
Caso M.E. 1979. Los Equinodermos de la Bahía de Mazatlán, Sinaloa. An. Cent. Cienc. Mar y Limnol. UNAM. México. 6: $197-368$.

Clark H.L. 1910. The Echinoderms of Peru. Bulletin of the Museum of Comparative Zoology at Harvard University. 52(17):321-358.

Clark A.M. 1993. An index of names of recent Asteroidea, part 2: Valvatida, in: Jangoux, M.; Lawrence, J.M. (Ed.) (1993). Echinoderm Studies, 4: pp. 187-366

Deichmann E. 1941. The holothuroidea collected by the Velero III during the years 1932 to 1938 . Part I. Dendrochirota. All. Hanc.Pac.Exped., 8(3):61-195.

Deichmann E. 1958. The Holothuroidea collected by the Velero III and IV during the years 1932 to 1954. Part. II Aspidochirota. All.Hanc.Pac. Exped., 11(2):253-348.

Gil D. \& H. Zaixso, 2008. Feeding ecology of the subantarctic sea star Anasterias minuta within tide pools in Patagonia, Argentina. Rev. Biol. Trop. Vol. 56 (Suppl. 3): 311-328

Himmelman J., C. Dutil \& C. Gaymer. 2005. Foraging behavior and activity budgets of sea stars on a subtidal sediment bottom community. J. Exp. Mar. Biol. Ecol. 322: 153-165.
Hooker Y., Solís-Marín F.A. \& M. Lleellish. 2005. Equinodermos de las Islas Lobos de Afuera (Lambayeque, Perú). Rev. Peru.Biol. 12(1):77-82.

Howell K., D. Pond, D. Billett, \& P. Tyler, 2003. Feeding ecology of deep-sea seastars (Echinodermata: Asteroidea): a fatty-acid biomarker approach. Marine Ecology - Progress Series, 255, 193-206.

Mutschke E. \& C. Mah, 2009. Asteroideos-Estrellas de Mar. En: Háussermann, V., G. Fórsterra (eds). Fauna Marina bentónica de la Patagonia Chilena, guía de identificación ilustrada. Nature in Focus, Chile. 801-830 p.

Prieto-Ríos E. 2010. Taxonomía de Holothuroidea (Echinodermata) del mar del Perú. Tesis para optar el Titulo Profesional. Facultad de Ciencias Biológicas. Escuela Académico Profesional de Ciencias Biológicas. Universidad Nacional Mayor de San Marcos, Lima, Peru. 71 p.

Rowe F. 1977. A new family of Asteroidea (Echinodermata), with the description of five new species and one new subspecies of Asterodiscides. Records of the Australian Museum 31(5): 187-233. 